\title{
Les Gekkonidae du Cameroun, avec la description de deux sous-espèces nouvelles par
}

\author{
J.L. PERRET \\ Muséum d’Histoire naturelle, Genève \\ Avec 5 figures dans le texte
}

L'étude des Gekkonidae de mes collections camerounaises, dẻposées au Muséum de Genève, ainsi que la revision des formes de cette famille, reconnues au Cameroun, m'amène à établir une liste de 18 espèces ou sous-espèces comprenant deux races nouvelles et deux espèces nouvelles pour le Cameroun.

\section{Cnemaspis spinicollis (L. Müller)}

Matériel: lacune.

Comme l'ont relevé Loveridge (1947) et Grandison (1956), il est évident que le genre Ancylodactylus est synonyme de Cnemaspis.

Je n'ai pas récolté $C$. spinicollis dans la région forestière du Sud Cameroun mais l'espèce a été retrouvée plus à l'ouest, jusqu'en Côte-d'Ivoire (coll. V. Aellen et British Museum), (Pasteur, 1961).

\section{Cnemaspis africanus köhleri Mertens}

Matériel: lacune.

Cette espèce, décrite de Buéa, Mont-Cameroun, n'a pas été retrouvée et provient d'une région favorable à l'endémisme. 
3. Cnemaspis quattuorseriatus dilepis ssp. n.

Matériel: 1 ^̊, holotype, Mus. Genève, 1005.69, de Foulassi, Sud Cameroun (forêt). Fig. 1.

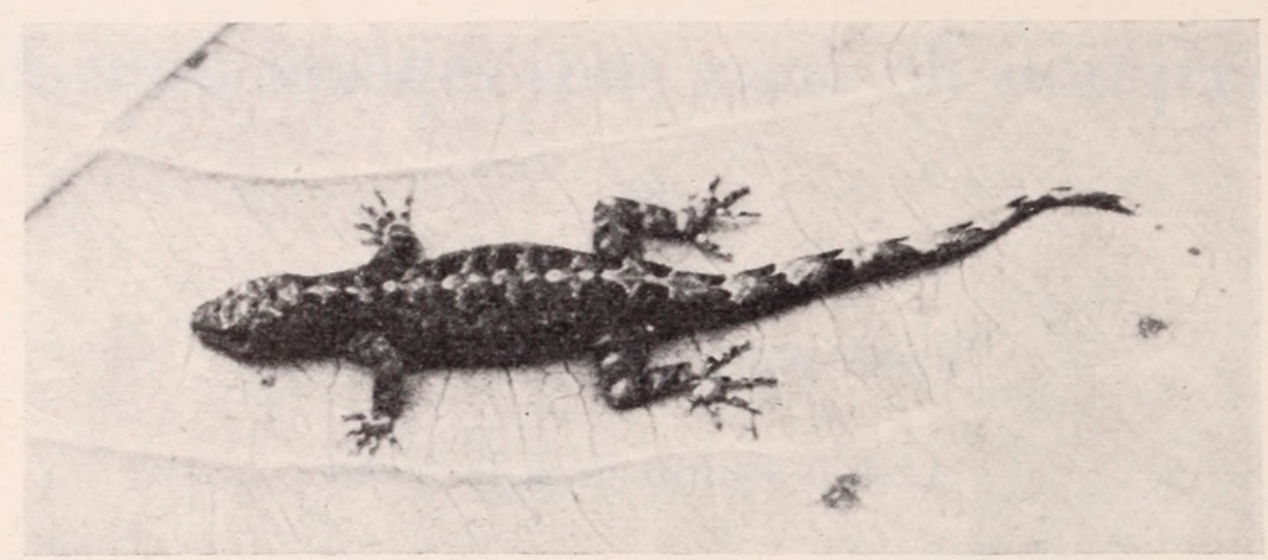

FIG. 1.

Cnemaspis quattuorseriatus dilepis ssp. n. Holotype $\widehat{\jmath}$. Grand. nat.

Jusqu'ici, C. quattuorseriatus n'était connu que de l'Afrique orientale (région des Grands Lacs). Mon exemplaire ne peut se rapporter à la race dickersoni Schmidt, d'ailleurs reconnue comme synonyme de quattuorseriatus par Loveridge (1947) parce qu'il possède bien quatre rangs de tubercules dorsaux. Il diffère essentiellement de la forme typique par la présence de 2 plaques sous l'orteil médian (au lieu de 3-5 plaques, normalement 4). Je le désigne comme holotype d'une race occidentale nouvelle qui sera confirmée ou non par des récoltes ultérieures.

\section{Diagnose:}

Rostrale plus large que haute, fissurée supérieurement. Narine entourée par la rostrale et par 3 nasales. Supranasales très larges, séparées par 2 granules. Symphysiale (mentonnière) très grande, bordée postérieurement par 4 sublinguales (postmentonnières), les deux externes les plus grandes. 5 labiales supérieures; 5 à 6 labiales inférieures. 2 plaques seulement sous la phalange basale de l'orteil médian. Séries transversales de ventrales: 22.8 pores préanaux en série continue. 
Coloration sur le vivant:

Dessus de la tête beige, tacheté de brun. Dessus du corps et de la queue brun foncé avec une série vertébrale de taches claires plus ou moins en forme d'étoile à quatre branches. Il y a 9 taches sur le corps de l'occiput au niveau anal et 6 à 7 sur la queue. Les petits tubercules dorsaux sont blancs.

La face inférieure porte trois couleurs distinctes: bleu acier sous la tête et le cou, jaune pâle, blanchâtre sous la poitrine et le ventre et orangé vif sous la queue, cette dernière couleur tranchant nettement avec la coloration ventrale claire, juste au niveau de l'anus.

Dans l'alcool, les colorations originales gulaire et subcaudale ont disparu et la face inférieure est uniformément jaunâtre.

Mensurations du type (en $\mathrm{mm}$ )

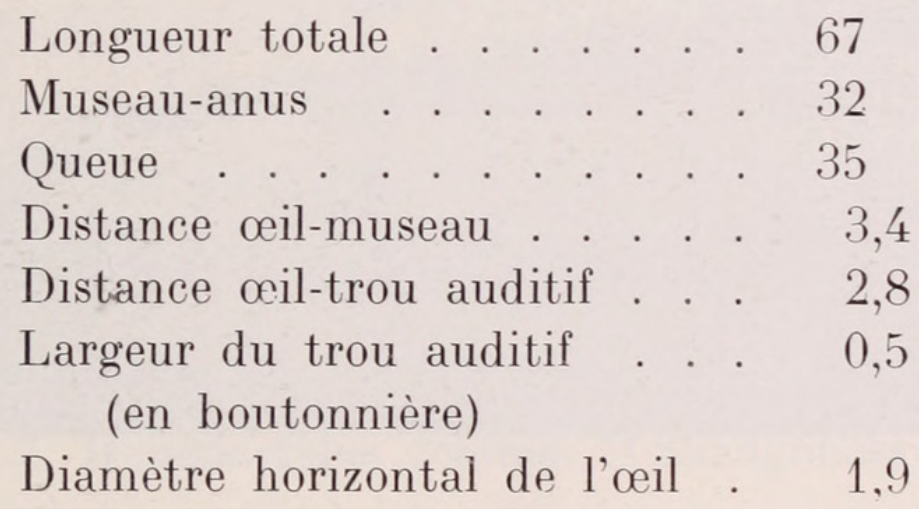

\section{Diplodactylus palmatus (Mocquard)}

Matériel: đo et + Mus. Genève, 1006.67-68, de Foulassi.

Ce remarquable gecko arboricole a été rarement capturé parce que sa présence près du sol est accidentelle. Je ne trouve que 9 labiales supérieures (type: 10-12); 8 labiales inférieures (type: 9-10), mais autrement il y a parfaite concordance avec la description de Mocquard. Loveridge (1947), dans sa redescription, commet l'erreur d'indiquer une rostrale fissurée («with a median cleft») alors que cette écaille n'est que faiblement concave supérieurement; il parle ensuite d'un disque adhésif à l'extrémité de la queue alors qu'il s'agit d'un groupe d'écailles plus grandes que les autres 
sous-caudales; il reproduit enfin, pl. II, fig. 15, le dessin de cette extrémité caudale donné par MüLLER (1910) mais en la désignant comme un détail du doigt chez Diplodactylus!

\section{Coloration sur le vivant:}

Dessus de la tête, côté de la tête et du corps, dessus de la moitié distale de la queue, gris brun foncé. Dessus du cou, du dos et de la moitié proximale de la queue présentant une alternance de 10 larges taches blanches sublozangiques et de 9 fines lignes brunes transversales irrégulières.

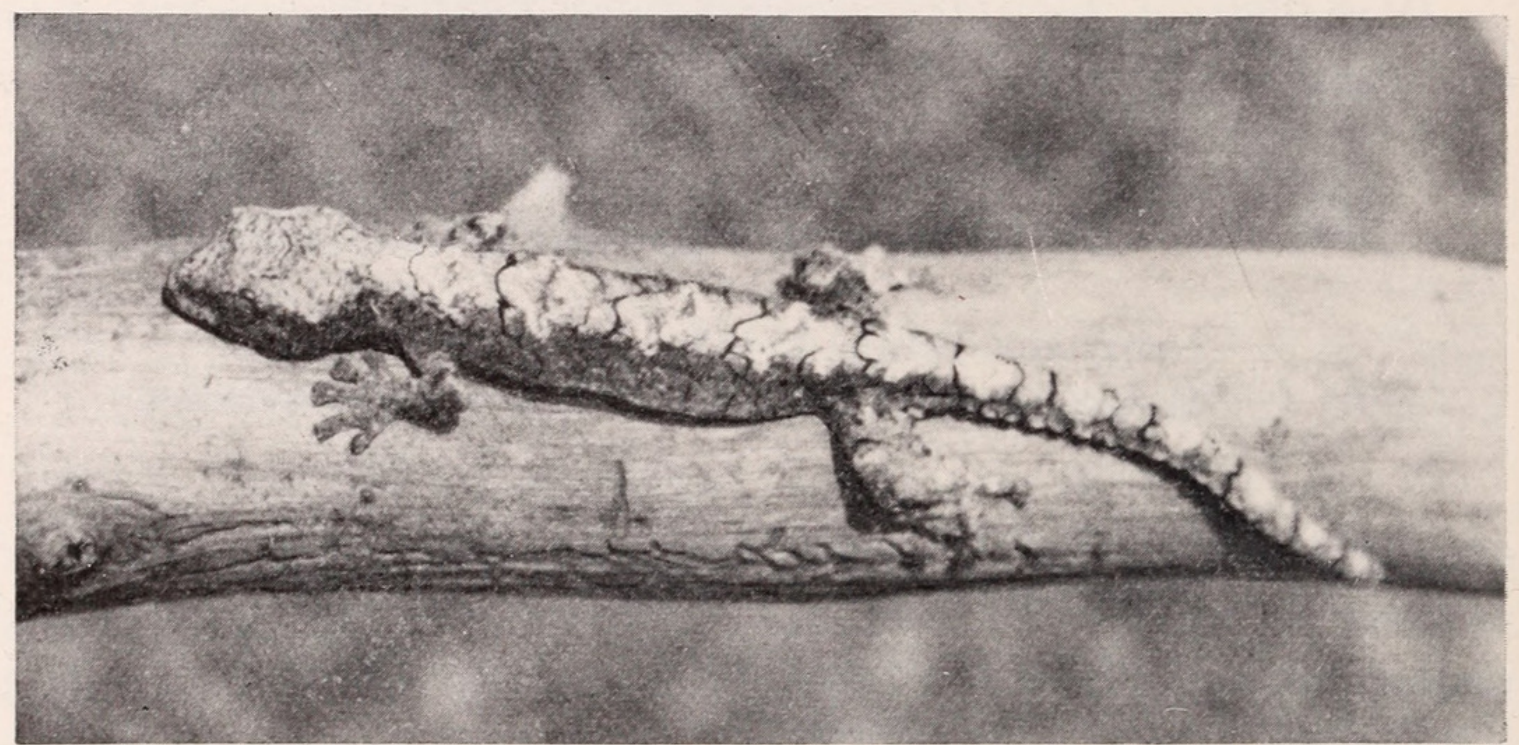

FIG. 2.

Diplodactylus palmatus (Mocquard). Grand. nat.

Chez mon deuxième exemplaire (ô), fig. 2, le dessus de la tête est complètement clair et les taches blanches vertébrales sont noyées dans une large zone blanche à gris très clair, allant de l'occiput à l'extrémité de la queue.

Face inférieure assez pigmentée, finement tachetée même à la périphérie, avec une tache jaune vif. préanale, très caractéristique. Une tache jaune moins nette, allongée, située entre les membres antérieurs, présente chez un exemplaire.

Précisons encore que le ô ne semble pas avoir de pores préanaux et que la paupière de $D$. palmatus est oblique et se ferme en haut en avant. 


\section{Diplodactylus weileri L. Müller}

Matériel: lacune.

Cette espèce n'a pas été retrouvée et sa synonymie avec palmatus a été soupçonnée. Cependant, Mertens (1938) a comparé le type de weileri avec du matériel de palmatus de même origine (matériel de Sјöstedt du Cameroun occidental, déterminé Phyllodactylus porphyreus) et l'a trouvé distinct. Il faut donc maintenir pour l'instant cette espèce camerounaise.

\section{Hemidactylus brooki angulatus Hallowell}

Matériel: 19 exemplaires.

ㅇ 917.52 et o 917.53 de Foulassi 1952; ơ 963.63 de Ngam/Sangmelima 1956; ỗ 1004.86-94 et 우 1004.95-100, 1005.1 de Ngaoundéré, Mbé et Maroua 1961.

Espèce très commune dans les savanes du Nord Cameroun mais existant également en forèt du sud (Foulassi), où elle semble beaucoup moins fréquente et légèrement différente par la coloration (Perret et Mertiens, 1957).

\section{Hemidactylus echinus O'Shaughnessy}

Matériel: 1 q Mus. Genève 918.45, de Tiko, Cameroun occidental, 1937 (éch. Senckenberg).

$\mathrm{Au}$ Cameroun, cette espèce n'est connue que de la région occidentale et de la plaine côtière.

\section{Hemidactylus f. fasciatus Gray}

Matériel: 23 exemplaires.

우 917.57-58 de Foulassi 1955; 우 963.64 et ô 963.65 et juv. 963.66-67 de Foulassi 1957; ỗ 10 1005.52-59 et 우우 1005.60-64 et juv. 1005.65-68 de Foulassi 1960-1961.

Espèce répandue en région forestière. En partie commensale de l'homme. 


\section{Hemidactylus longicephalus Bocage}

Matériel: 24 exemplaires.

ôo 963.72-74 et 우 963.77-78 et juv. 963.79-80 de Ngam Sangmelima 1957; ô 963.75 et \% 963.76 de Bangwa 1957; ô 1005.3 de Douala 1959; 우 1005.4-12 et ỗ 10 1005.13-17 de Foulassi 1960-1961.

Espèce fréquente en région forestière du Sud Cameroun. La synonymie avec $H$. steindachneri Tornier me semble pleinement confirmée.

\section{Hemidactylus mabouia (Jonnès)}

Matériel: 20 exemplaires.

ô 963.68 de Nkongsamba 1956; qo 963.69-70 de Yaoundé 1957; ô 963.71 de Douala 1958; ỗ $1004.80-85$ et o 1005.18 et juv. 1005.19-25 de Yaoundé 1961.

Cette forme est bien répandue dans les villes du Sud Cameroun, surtout Douala et Yaoundé, où elle hante les habitations. Loverid GE (1947) la croit introduite en Afrique occidentale. Le commensalisme urbain de l'espèce, au Cameroun, le laisse en effet supposer.

\section{Hemidactylus muriceus Peters}

Matériel: 1 đ 918.53, de Mubenge, Cameroun occidental 1937; (éch. Senckenberg).

$\mathrm{Au}$ Cameroun cette espèce n'est connue que de la région occidentale et de la plaine côtière, comme echinus.

\section{Hemidactylus richardsoni (Gray)}

Matériel: 28 exemplaires.

우 917.54-55 de Foulassi 1953; ô 9 963.81-84 et o+ 963.85-86 de Foulassi 1957; ô ô 1005.32-40 et 우 1005.41-47 et juv. 1005.48-51 de Foulassi 1960-1961.

Espèce très commune en région forestière du Sud Cameroun; prolifère dans les cases des villages habite aussi les souches des vieux arbres. 


\section{Lygodactylus angularis dysmicus ssp. n.}

Holotype: đo Mus. Genève 1005.72, provenant de Foulassi, Sud Cameroun forestier, à la limite nord-occidentale du bassin du Congo 9.1961. Fig. 3.

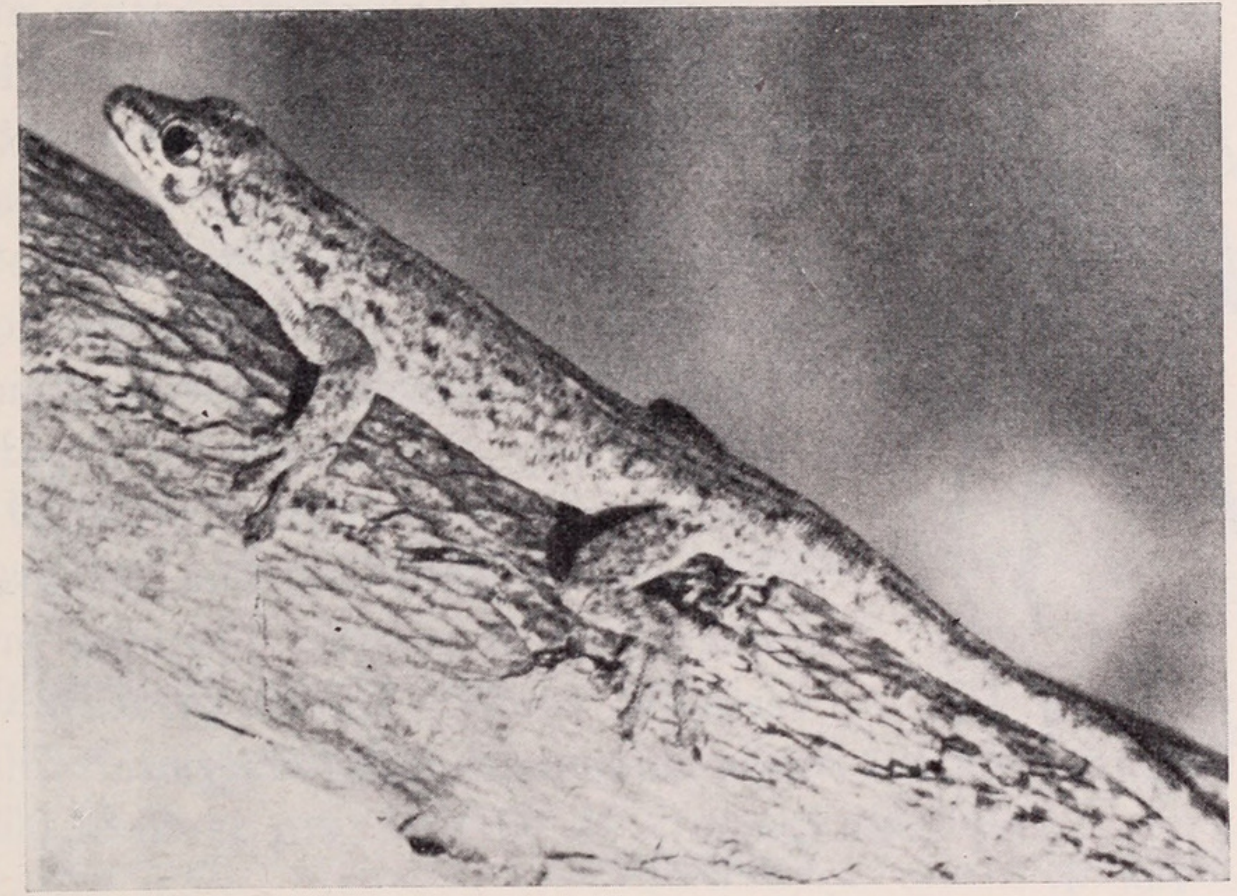

Fig. 3.

Lygodactylus angularis dysmicus ssp. n. Holotype ô. Gross. $2 \times$.

\section{Diagnose:}

Race occidentale de $L$. angularis correspondant par la pholidose aux deux formes déjà décrites, $a$. angularis et $a$. heeneni, de l'Afrique orientale et du Katanga, mais différant d'elles, principalement, par l'ornementation gulaire qui se compose de deux taches en forme d'U, l'une dans l'autre, et d'une marque centrale (fig. 4). Ces taches en U, subparallèles, ouvertes en arrière, se rapprochent $\mathrm{du}$ style gulaire de certains $L$. picturatus ou thomensis mais sont le contraire des lignes convergeant en arrière des angularis orientaux. En outre la tête de dysmicus possède des particularités, non signalées chez les races orientales: une grosse tache foncée arrondie à la commissure des lèvres, une ligne foncée brisée, transversale, joignant les yeux à leur niveau antérieur et une tache foncée médiane sur le museau. 


\section{Description :}

Museau assez large, sa longueur (œil-bout du museau) étant égale à l'écart interorbital antérieur mais notablement plus grande que la distance entre l'œil et le trou auditif. Celui-ci petit elliptique à grand axe vertical. De diamètre vertical à peu près égal à la longueur de la troisième ou de la quatrième labiale supérieure. Narine

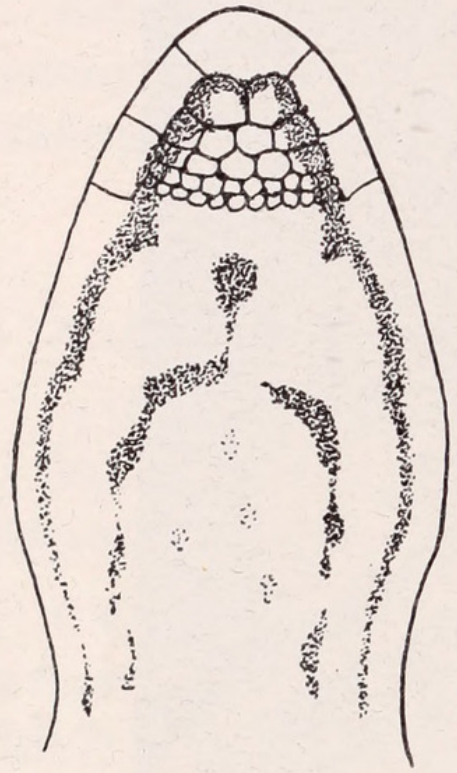

FIG. 4.

Ornementation gulaire chez Lygodactylus angularis dysmicus.

horizontalement elliptique, située au-dessus de la suture entre la rostrale et la première labiale supérieure, en contact avec ces écailles ainsi qu'avec trois nasales. 2 granules internasaux. Symphysiale (mentonnière) entière, plus large que longue, suivie de 2 sublinguales (postmentonnière. 7 labiales sup., 6-7 labiales inf. 5 paires de lamelles sous le quatrième orteil. 9 pores préanaux, en série continue. 220 granules dorsaux, de la rostrale jusqu'au premier verticille caudal. Queue présentant une série de sous-caudales élargies.

\section{Coloration :}

Dorsalement gris-brun; une raie canthale brun foncé se poursuivant derrière l'œil jusqu'au membre antérieur; une grande tache foncée à la commissure des lèvres; une ligne brisée transversale reliant les yeux à leur niveau antérieur; une tache foncée médiane, sur le museau; quelques taches étroites sur la tête; trois à quatre lignes foncées, légèrement en zig-zag, de chaque côté du cou; toute la surface dorsale mélangée de brun sur fond gris, sur lequel se détachent deux rangées de taches foncées, doublées de taches blanches. Ces rangées courant de chaque côté du dos, de la région occipitale à la naissance de la queue.

Dessous clair (animal fixé) sauf la moitié distale de la queue qui est grise et la face inférieure de la tête qui est ornée de deux taches en forme d'U ouvert en arrière; la plus grande tache en $\mathrm{U}$ borde les labiales inférieures et la symphysiale; une tache centrale enfin est reliée plus ou moins distinctement par un isthme à la petite tache en $\mathrm{U}$ et forme avec elle une image de diapason. 
Sur le vivant, la coloration est blanche sous la tête et jaune sous le ventre et les membres.

\section{Mensurations du type (en $\mathrm{mm}$ )}

Longueur totale . . . . . . 57

Longueur de la queue . . . 28,5

Longueur museau-anus . . . . 28,5

Distance œil-museau . . . . 4

Distance interorbitale . . . 4

Distance trou auditif-œil . . . 2,8

Hauteur du trou auditif . . . 0,8

Diamètre horizontal de l'œil . . 2

\section{Lygodactylus c. conraui Tornier}

Matériel: 18 exemplaires.

ㅇ 963.87 et ơ 963.88 de Ngam et Foulassi 1956-57; ô ơ1006.51-57, 우 1006.58-65 et juv. 1006.66 de Foulassi 1960-1961.

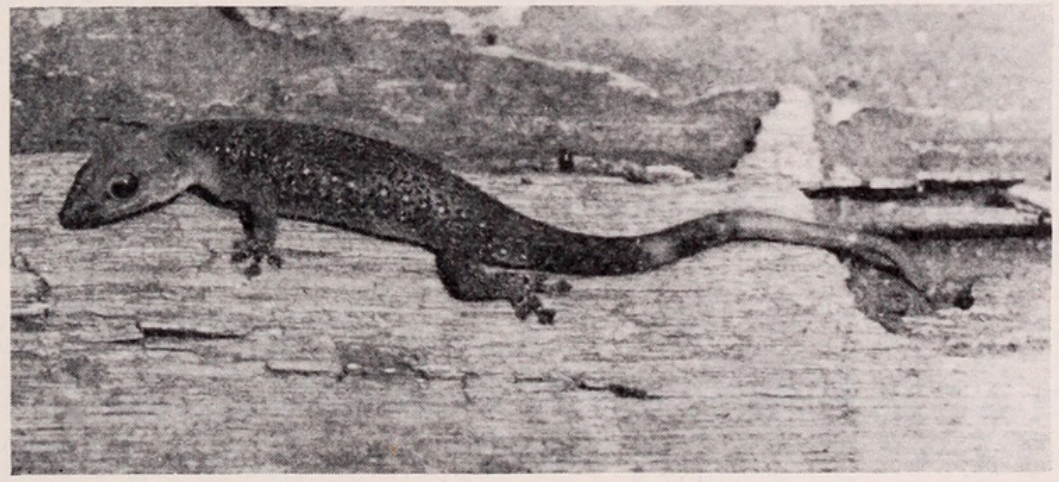

F1G. 5 .

Lygodactylus conraui conraui Tornier.

Remarquer le double rang latéral de taches ocellées. Grand. nat.

Le matériel récolté en Côte-d'Ivoire par V. Aellen, représente une race qui présente des différences de coloration constantes et nettes, en particulier l'absence de rangées latérales de taches ocellées à centre bleu et bord foncé, si caractéristique de la forme typique du Cameroun. Sur le vivant, la coloration de la tête et du corps 
est verte, la queue étant jaune orangé. Parfois la coloration orangée envahit aussi la tête et dans ce cas le corps seul est vert. Sur ce fond vert, le corps est finement tacheté de noir, les côtés sont ornés de deux rangées de taches ocellées noires à centre bleu (et non d'une seule, Tornier 1902) (fig. 5). A noter que souvent, chez les exemplaires fixés, tous ces détails pâlissent, disparaissent même, surtout chez les jeunes, l'animal devenant gris-brun uniforme.

Ce petit gecko diurne, arboricole, est encore rare en collection, aussi je crois utile de donner un tableau analytique groupant les variations de quelques caractères observés sur les 18 spécimens en ma possession.

Variations chez Lygodactylus conraui conraui Tornier du Cameroun.

\begin{tabular}{|c|c|c|c|c|c|c|c|c|c|}
\hline 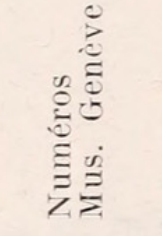 & $\begin{array}{l}\stackrel{u}{u} \\
0 \\
0\end{array}$ & 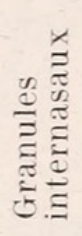 & 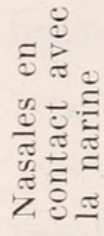 & 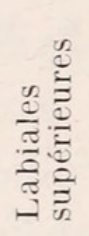 & 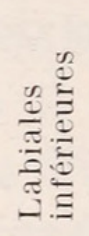 & 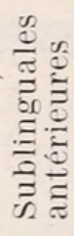 & 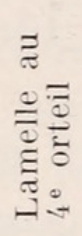 & 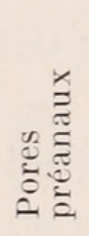 & 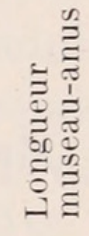 \\
\hline 963.88 & $\hat{0}$ & 2 & 3 & 7 & 8 & 3 & 5 & 4 & 25 \\
\hline 1006.51 & $\hat{0}$ & 2 & 3 & 7 & 8 & 2 & 5 & 4 & 31 \\
\hline 1006.52 & 0 & 2 & 3 & 7 & 7 & 3 & 5 & 4 & 24 \\
\hline 1006.53 & 0 & 2 & 2 & 7 & 7 & 3 & 5 & 4 & 26 \\
\hline 1006.54 & 0 & 3 & 3 & 7 & 8 & 3 & 5 & 4 & 27 \\
\hline 1006.55 & $0 \hat{0}$ & 2 & 3 & 7 & 7 & 3 & 5 & 4 & 28 \\
\hline 1006.56 & o & 2 & 3 & 8 & 7 & 3 & 5 & 4 & 21 \\
\hline 1006.57 & o & 3 & 3 & 7 & 8 & 3 & 5 & 4 & 23 \\
\hline 963.87 & 우 & 2 & 3 & 8 & 7 & 3 & 5 & - & 25 \\
\hline 1006.58 & q & 3 & 3 & 8 & 7 & 3 & 5 & 一. & 30 \\
\hline 1006.59 & q & 1 & 3 & 7 & 8 & 3 & 5 & - & 27 \\
\hline 1006.60 & 우 & 1 & 3 & 8 & 7 & 3 & 5 & - & 26 \\
\hline 1006.61 & 우 & 2 & 3 & 8 & 7 & 3 & 5 & - & 30 \\
\hline 1006.62 & 우 & 3 & 3 & 7 & 7 & 3 & 5 & - & 24 \\
\hline 1006.63 & 우 & 2 & 3 & 7 & 7 & 3 & 5 & 一 & 29 \\
\hline 1006.64 & 우 & 2 & 3 & 7 & 8 & 3 & 5 & - & 27,5 \\
\hline 1006.65 & 우 & 2 & 3 & 7 & 8 & 3 & 5 & - & 29,5 \\
\hline 1006.66 & juv. & 3 & 3 & 7 & 7 & 3 & 5 & - & 18 \\
\hline
\end{tabular}

De ce tableau, on peut tirer la formule qui représente la tendance normale chez cette forme, elle donne, dans l'ordre des caractères mentionnés:

$$
2-3-(7-8)-(7-7)-3-5-4
$$




\section{Lygodactylus fischeri Boulenger}

Matériel: 2 exemplaires.

ô ô 1005.70-71 de Foulassi, Sud Cameroun 1960-1961.

Il est également possible que cette espèce décrite de Sierra Leone soit représentée par une race au Cameroun. Mes deux exemplaires correspondent bien à la description de Loveridge (1947), sauf deux caractères d'ailleurs peu importants, indiqués dans le tableau par un point exclamatif.

\begin{tabular}{|c|c|c|c|}
\hline & $\begin{array}{c}\text { LOVERIDGE } \\
1947\end{array}$ & Ex. 1005.70 & Ex. 1005.71 \\
\hline 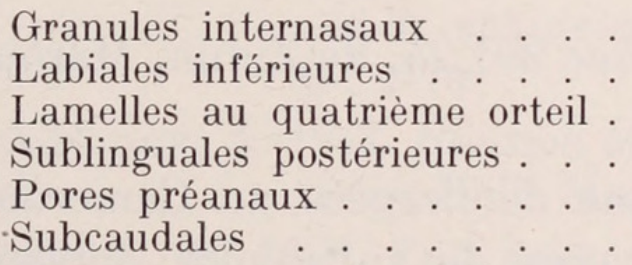 & $\begin{array}{c}2-3 \\
6-9 \\
\text { 4-5 paires } \\
3 \\
7-10 \\
\text { paires }\end{array}$ & $\begin{array}{c}3 \\
7-8 \\
5 \\
2 ! \\
9 \\
\text { paires }\end{array}$ & $\begin{array}{c}2 \\
7 \\
5 \\
3 \\
8 \\
\text { mixtes ! }\end{array}$ \\
\hline
\end{tabular}

La coloration correspond à la description des auteurs sauf que les grandes taches noires au-dessus des avant-bras, signalées sur le matériel camerounais, manquent chez mes deux exemplaires.

\section{Ptyodactylus h. hasselquisti (Donndorf)}

\section{Matériel: 6 exemplaires}

ô ô 1005.26-27, 우 1005.28-30 et juv. 1005.31 de Zidim, Maroua, Nord Cameroun 1961.

Le gecko des tombeaux n'avait jamais été signalé au Cameroun jusqu'ici ni à une latitude si méridionale. Il m'a semblé assez fréquent à Maroua qui se trouve à $270 \mathrm{~km}$ environ au sud du lac Tchad (savane soudanienne).

\section{Tarentola annularis (Geoffroy)}

Matériel: 23 exemplaires.

991.1-23 de Zidim Maroua, Nord Cameroun 1961. 
A. G. C. Grandison (1961) a mis au point le statut de cette espèce et a confirmé, après examen, l'identification de la présente série d'annularis ainsi que celle d'un unique exemplaire ephippiata provenant du même lieu.

Le caractère le plus saillant (à part la coloration), pour distinguer annularis d'ephippiata dans ce matériel, est celui des écailles gulaires, plus fines et plus nombreuses chez annularis. Par contre, les tubercules ovalaires dorsaux chez les exemplaires adultes d'annularis sont tout à fait lisses sur les trois quart du dos et la présence d'une carène n'est donc pas un caractère vraiment distinctif.

\section{Tarentola ephippiata O'Shaughnessy}

Matériel: 1 exemplaire Mus. Genève 991.24, de Zidim, Maroua, Nord Cameroun 1961.

C'est la première capture certaine de l'espèce au Cameroun. Cet exemplaire possède quatorze rangs de tubercules dorsaux, minimum chez l'espèce (Grandison, 1961).

Résumé des synonymes et espèces erronnées.

Les anciennes listes de Sauriens du Cameroun de Nieden (1910) et de Monard (1951), renferment des espèces tombées en synonymie. Ce sont Hemidactylus steindachneri Tornier qui est devenu H. longicephalus Bocage, $H$. stellatus Boul. devenu $H$. brooki angulatus Hallowell, Tarentola senegalensis Boul. devenu T. ephippiata O'Shaughnessy. Il faut en outre retrancher de ces listes les espèces erronées: Phyllodactylus porphyreus (Daudin) et Stenodactylus elegans mauritanica Guichenot. C'est à Tornier (1902) qu'on doit la citation qui ne peut être qu'erronée de cette dernière espèce au Cameroun, à Bipindi, localité du Sudf orestier. Cependant, après ma découverte de Ptyodactylus à Maroua, on peut se demander, si un Stenodactylus pourrait également atteindre l'extrême Nord du Cameroun, les deux genres se trouvant ensemble jusqu'en Aïr (ANGEL, 1950). 


\section{BIBLIOGRAPHIE}

Angel, F. 1930. Reptiles et Batraciens recueillis par M. Monod au Cameroun. Bull. Mus. Hist. nat. Paris 2: 253-254.

- 1943. Sur deux Lézards nouveaux de la Haute-Guinée française, appartenant aux familles des Amphisbaenidés et des Gekkonidés. (Matériaux de la Mission Lamotte, au Mont-Nimba, en 1941). Première Note. Bull. Mus. Hist. nat. . aris 15: 163-166.

- 1950. Lézards. In: Contribution à l'étude de l’Ä̈r. Mém. Inst. franç. Afr. noire 10: 331-336.

Grandison, A. G. C. 1956. On a collection of lizards from West Africa. Bull. Inst. franç. Afr. noire 18 A: 224-245.

- 1961. Preliminary notes on the taxonomy of Tarentola annularis and T. ephippiata (Sauria: Gekkonidae). Zool. Meded. 38: 1-14.

Loveridge, A. 1947. Revision of the African lizards of the family Gekkonidae. Bull. Mus. comp. Zool. Harvard 98: 1-469.

Mertens, R. 1937. Eine neue, tiergeographisch bemerkenswerte Eidechse aus Kamerun. Senckenbergiana 19: 381-384.

1938. Herpetologische Ergebnisse einer Reise nach Kamerun. Abh. senckenberg. naturf. Ges. 442: 1-52.

Monard, A. 1951. Résultats de la mission zoologique suisse au Cameroun. 4. Reptiles. Mém. Inst. franç. Afr. noire Centre du Cameroun, sér. Sci. nat. 1: 123-170.

Müller, L. 1907. Über einen neuen Gecko aus Kamerun und eine neue colubrine Schlange aus Centralchina. Zool. Anz. 31: 824-830.

- 1909. Vorläufige Mitteilung über ein neues Chamäleon und einen neuen Gecko aus Kamerun. Wiesbaden Jahrb. Ver. Natk. 62: 111-115.

1910. Beiträge zur Herpetologie Kameruns. Abh. bayer. Akad. Wiss. math. phys. Kl. 24: 543-626.

Nieden, F. 1910. Die Reptilien (ausser den Schlangen) und Amphibien. In: Die Fauna der deutschen Kolonien I. Kamerun, H. 2. Berlin: 1-74.

Pasteur, G. 1961. Note préliminaire sur les geckos rapportés de la Côte d'Ivoire par V. Aellen. C.R. Soc. Sci. nat. phys. Maroc 27: 105.

Perret, J.-L. et R. Mertens. 1957. Etude d'une collection herpétologique faite au Cameroun de 1952 à 1955. Bull. Inst. franç. Afr. noire 19 A: 548-601. 
Schmidt, K. P. 1919. Contributions to the herpetology of the Belgian Congo, based on the collection of the American Museum Congo Expedition, 1909-1915. Part I. Turtles, Crocodiles, Lizards, and Chamaeleons. Bull. amer. Mus. nat. Hist. 39 : 385-624.

Tornier, G. 1902. Die Crocodile, Schildkröten und Eidechsen in Kamerun. Zool. Jahrb., Syst. 15: 663-677.

Witte, G. F. De. 1941. Batraciens et Reptiles. In: Exploration du Parc National Albert Mission G. F. de Witte (1933-1935) 33. Inst. Parcs nat. Congo belge, Bruxelles: I-XVII, 1-261. 


\section{$2 \mathrm{BHL}$ Biodiversity Heritage Library}

1963. "Les Gekkonidae du Cameroun, avec la description de deux sous-especes nouvelles." Revue suisse de zoologie 70, 47-60. https://doi.org/10.5962/bhl.part.117919.

View This Item Online: https://www.biodiversitylibrary.org/item/149506

DOI: https://doi.org/10.5962/bhl.part.117919

Permalink: https://www.biodiversitylibrary.org/partpdf/117919

\section{Holding Institution}

American Museum of Natural History Library

\section{Sponsored by}

BHL-SIL-FEDLINK

\section{Copyright \& Reuse}

Copyright Status: In copyright. Digitized with the permission of the rights holder.

Rights Holder: Muséum d'histoire naturelle - Ville de Genève

This document was created from content at the Biodiversity Heritage Library, the world's largest open access digital library for biodiversity literature and archives. Visit BHL at https://www.biodiversitylibrary.org. 